\title{
Etats, minorités et diasporas. Réflexions à propos de l'ouvrage de Basma Kodmani-Darwish
}

Rémy Leveau et Nikola Tietze

\section{(2) OpenEdition \\ 1 Journals}

Édition électronique

URL : http://journals.openedition.org/conflits/543

DOI : $10.4000 /$ conflits. 543

ISSN : $1777-5345$

Éditeur :

CCLS - Centre d'études sur les conflits lilberté et sécurité, L'Harmattan

Édition imprimée

Date de publication : 15 mai 1998

ISSN : 1157-996X

Référence électronique

Rémy Leveau et Nikola Tietze, «Etats, minorités et diasporas. Réflexions à propos de l'ouvrage de Basma Kodmani-Darwish», Cultures \& Conflits [En ligne], 31-32 I printemps-été 1998, mis en ligne le 16 mars 2006, consulté le 30 mars 2021. URL : http://journals.openedition.org/conflits/543; DOI : https://doi.org/10.4000/conflits.543

Ce document a été généré automatiquement le 30 mars 2021.

Creative Commons License 


\title{
Etats, minorités et diasporas. Réflexions à propos de l'ouvrage de Basma Kodmani-Darwish
}

\author{
Rémy Leveau et Nikola Tietze
}

1 L'ouvrage de Basma Kodmani-Darwish ${ }^{1}$ donne enfin une présentation en langue française claire, globale et équilibrée de la question palestinienne mais offre également une base de réflexion sur les rapports entre Etats, minorités et diasporas au MoyenOrient. Sans négliger la dimension historique, l'auteur concentre son analyse sur les conséquences de la territorialisation du projet palestinien à la suite des accords d'Oslo et de Washington de septembre 1993. Son ouvrage présente à la fois un récit des événements, un tableau des situations et un questionnement sur l'avenir, à partir du constat d'autonomie limitée sur un territoire manquant de consistance. Le mouvement palestinien s'incarne alors dans une sorte de sionisme inversé et tronqué, où Arafat joue, dans un autre registre, le rôle de Ben Gourion.

2 Mais, de façon plus large, l'ouvrage pose le problème d'une recomposition du système moyen-oriental qui ne serait pas uniquement fondée sur le principe de la souveraineté des Etats, mais prendrait largement en compte la protection des minorités. Dans le cas palestinien, le changement de rôle par rapport à une stratégie de l'OLP qui s'appuyait sur la diaspora redonne à cette dernière un destin à construire là où elle s'est installée. Progressivement, les responsables de l'Autonomie vont perdre la possibilité de parler au nom des Palestiniens de l'extérieur et ces derniers vont devoir assumer les compromis à passer avec les pouvoirs arabes là où ils sont installés. La diaspora existait en fait depuis longtemps, mais elle va se trouver conduite à prendre en charge son destin tout en maîtrisant les conséquences de ses solidarités extérieures.

Par voie de conséquence, c'est par la mise en place d'un système régional sous contrôle international que l'on peut espérer combiner sécurité, prospérité et soutiens extérieurs pour stabiliser ce nouvel ensemble politique composé d'Etats, mais aussi de groupements d'intérêts collectivement reconnus que constituent les minorités. Travailler dans un milieu où la plupart des acteurs n'ont pas vraiment renoncé à la 
violence rend la chose encore plus complexe. Seuls quelques groupes culturels et identitaires ont réussi à se transformer en Etats-nations en créant un bouleversement plus grand que les pouvoirs impériaux ottomans, britanniques ou français. Mais ce nouvel ensemble régional reste à construire sans doute sous la pression d'acteurs extérieurs (Etats-Unis, Europe, Russie) qui ne voudront plus être impliqués - malgré eux - par des Etats ou des groupes sociaux non stabilisés et de ce fait engagés dans une recherche permanente d'appuis.

4 Pour s'y reconnaître dans ces stratégies, savoir à partir de quelles données se sont fabriquées des imaginaires collectifs excluant les solutions simples, l'ouvrage de Basma Kodmani-Darwish nous fournit des repères, des itinéraires et des analyses de cas. A l'origine des diasporas on retrouve toujours un traumatisme qui met en branle une population; dans le cas présent, les défaites arabes de 1948 et 1967. Ceux qui partent les premiers ont des moyens et des relations leur permettant de s'établir à Beyrouth ou à Amman. Puis, la guerre psychologique des groupes armés juifs et les violences visant à répandre la terreur font fuir un nombre plus important encore avant l'engagement des armées arabes dans le conflit. Au total, ce sont 800 à 900000 personnes qui deviennent des réfugiés. Il apparaît très vite que l'Etat d'Israël ne souhaite pas leur retour. Le provisoire s'installe avec la création d'une nouvelle administration des Nations-Unies, l'UNRWA, chargée tout spécialement des réfugiés de Palestine au dehors de la Croix Rouge et du H.C.R. Un second chapitre historique est consacré à l'analyse de l'OLP. La dispersion des réfugiés et le besoin de pérenniser la revendication territoriale donne naissance à une organisation construite sur le modèle des mouvements de libération des années soixante. Elle se fixe comme objectif de préserver un peuple en retissant les liens entre les groupes dispersés et en centrant sa revendication sur une terre à libérer. La création d'une identité palestinienne forte et d'un quasi-Etat sans territoire est mise au crédit de l'organisation. Le développement d'institutions, notamment pendant la période où l'OLP s'installe à Beyrouth, de 1970 à 1982, montre plus sa capacité à collecter et à redistribuer des ressources qu'à conduire des opérations militaires contre Israël. Pendant cette période, l'OLP doit aussi faire des choix entre une idéologie panarabe qui justifie les interventions dans les affaires intérieures des pays hôtes, comme la Jordanie et le Liban, et une stratégie aux objectifs territoriaux plus limités, œuvrant pour la création d'un petit Etat palestinien aux côtés d'Israël et renoncer de ce fait aux buts mythiques, sans perdre le sentiment d'appartenance commune.

5 Après ces chapitres historiques, l'auteur passe en revue différents cas d'installation, en commençant par la Jordanie. Ce pays offre le paradoxe d'avoir connu des périodes d'affrontements très violents avec l'OLP (septembre noir 1970) et de s'être montré, dans la longue durée, le plus accueillant aux Palestiniens, y compris en 1991, lorsqu'elle recueille 320000 d'entre eux expulsés du Koweït après la guerre du Golfe. La Jordanie est le seul pays qui ait donné des passeports nationaux aux Palestiniens, leur permettant d'avoir accès plus facilement au marché du travail des monarchies pétrolières, tout en leur assurant une intégration économique et politique locale. Vivant aussi bien dans les camps que dans les quartiers bourgeois, ceux-ci constituent aujourd'hui la majorité de la population du pays et imprègnent les villes de leur culture et de leur savoir-faire. A la fois contrôlés et intégrés, ils n'ont pu cependant développer un pouvoir autonome qui aurait mis en péril la monarchie. Si l'Intifada entraîne en 1988 la rupture de la fiction de l'unité avec la Cisjordanie, le souci principal du souverain après 1993 est d'éviter que les difficultés d'un règlement de la question palestinienne limité à Gaza et à la Cisjordanie n'ait des conséquences déstabilisantes 
pour son royaume. Cela explique à la fois son acceptation d'une paix prudente avec Israël en 1994 et son refus d'un lien fédéral avec l'Autonomie de crainte d'être trop directement impliqué comme solution de recours dans la mise en œuvre du processus initié par les accords d'Oslo. Par ailleurs, ces accords ont eu pour effet de dissocier les intérêts des Palestiniens de Jordanie et ceux de l'OLP. Leur identification au pays s'est accrue avec le souci de voir leurs intérêts représentés sans intermédiaires. Mais leur identité palestinienne ne peut s'accomplir que si l'Autonomie progresse de façon satisfaisante. La dimension affective et la mémoire collective particulièrement sensible à une reconnaissance du " droit de retour " par Israël militent donc en faveur d'une solution régionale s'appuyant sur des soutiens économiques extérieurs. Par opposition à la Jordanie, la situation des Palestiniens au Liban est dangereusement précaire. Après avoir tenu le haut du pavé face à l'Etat et aux communautés libanaises, à la suite des accords du Caire de 1969 qui leur concédaient une autonomie qui faisait de l'OLP un Etat dans l'Etat, et en fait un véritable pouvoir arbitral, ils ont subi l'invasion israélienne en 1982, les massacres de Sabra et Chatila, les attaques syriennes et la guerre des camps. Les accords de Taef en 1989 ne mentionnent pas leur présence et les accords d'Oslo en 1993 ont entraîné chez les Libanais une réaction unanime de rejet d'une intégration qui risquerait de bouleverser l'équilibre communautaire. Les élites palestiniennes ont quitté le Liban lors de la guerre civile et de l'invasion israélienne. Il ne reste que les pauvres que les Libanais contrôlent étroitement et poussent au départ en leur interdisant la plupart des professions. Paradoxalement, c'est peut-être la protection syrienne ou le simple désir de maintenir un jeu complexe pour mieux le contrôler qui empêche aujourd'hui la mise en œuvre d'une exclusion sur une grande échelle. La Syrie a en revanche pratiqué au cours des années une forme d'intégration des Palestiniens inspirée par les principes du nationalisme arabe. Mais ce pays a en même temps exercé un contrôle politique strict, et s'est montré plutôt hostile à l'influence de l'OLP. L'intégration des Palestiniens dans l'armée syrienne a en fait permis à Damas d'exercer des pressions notamment sur les camps palestiniens au Liban. Après avoir constitué le groupe le plus prospère, l'élite de la diaspora, les Palestiniens du Koweït sont devenus après la guerre du Golfe des parias pourchassés, que la Jordanie a recueilli pour la plupart (320 000). Seront-ils une exception ou l'exemple d'épreuves qui pourraient toucher d'autres pays? En fait, leur sort était scellé bien avant l'invasion de l'Irak à partir du moment où leur proportion était jugée intolérable (400 000 contre 600000 Koweïtiens). Mais le Koweït sans les Palestiniens reste-t-il un Etat viable, et n'est-il pas encore plus artificiel qu'auparavant? L'auteur termine son tour d'horizon par l'évocation d'un groupe dont on parle peu: les Palestiniens israéliens qui dépasseront le chiffre de 900000 en l'an 2000. Les accords d'Oslo les conduisent à réaffirmer leur citoyenneté israélienne, tout en considérant dans leur majorité Israël à la fois comme un Etat illégitime et une réalité politique qui s'impose à eux. Ils cumulent de ce fait tous les paradoxes des minorités, réclamant à la fois l'égalité et un statut particulier. Bénéficiant marginalement des droits sociaux et de l'éducation du système israélien, ils ont pu développer de nouvelles couches d'élites intellectuellement à l'aise aussi bien dans le système israélien que dans l'espace international. Elles ont plus de difficultés à se faire admettre par les autres arabes. L'Intifada et les accords d'Oslo ont accru leur solidarité avec le reste de la diaspora sans les soumettre pour autant à l'OLP. Leur destin dépend en fait du type de citoyenneté que développera Israël. Si celle-ci reste fondée sur une base religieuse, les Palestiniens n'ont guère de place dans la construction de l'Etat, mais cela les conduira à user de leur 
capacité de nuisance à l'intérieur du système tout en évitant de se faire exclure. L'exercice risque d'être à la longue périlleux.

6 A la suite de cet itinéraire documenté à travers les lieux de la diaspora palestinienne, l'ouvrage aborde une série de questionnements. En premier lieu le problème des réfugiés, du droit de retour, qui a tenu une place si importante dans la formation de l'identité et de l'inconscient collectif des Palestiniens. Les accords d'Oslo font prendre conscience du caractère mythique de la revendication et l'OLP n'y tient guère plus aujourd'hui qu'Israël. Mais sans reconnaissance symbolique et politique, il ne peut y avoir renoncement ni mise en place d'une politique de compensation. Le vrai problème est l'insertion dans les sociétés d'accueil : mais celle-ci ne peut s'opérer qu'après une " catharsis " et des mesures pratiques de libre circulation à l'échelle régionale, entre les différents éléments de la diaspora qui sont encore éloignés aujourd'hui. La priorité est donnée depuis 1988 et surtout 1993 à la construction d'un espace national qui apparaît avec certes bien des différences comme une revendication parallèle à celle qui a conduit à la création d'Israël, en dépit de multiples contraintes. L'OLP doit après les accords d'Oslo à la fois élargir le contenu de l'Autonomie et s'assurer l'accès régulier au marché du travail israélien. La rivalité naturelle entre les élites locales de Cisjordanie et les cadres extérieurs de la résistance palestinienne risque d'être exploitée par Israël. A l'opposé, l'enracinement à Gaza et en Cisjordanie coupe progressivement de la diaspora une direction de l'OLP trop préoccupée de collecter des aides extérieures sans assurer pour autant une protection suffisante. Les camps, comme les bourgeois d'Amman, s'éloignent d'Arafat pour des raisons différentes. La diaspora ne peut plus se considérer comme l'extension d'un pouvoir tronqué, basé en Cisjordanie et à Gaza. Pour l'auteur, la création d'un Etat contribuera à accélérer et achever le processus de diasporisation (p. 181). Paradoxalement, les deux démarches sont complémentaires et concernent dans la région un archipel de groupements identitaires qui ne peuvent se contenter d'un seul mode de fonctionnement. La construction d'un Etat-nation reste un but auquel personne n'entend renoncer. Mais sa réalisation finale, ou son maintien, entraînent la perpétuation de la violence. L'entrecroisement de ces tensions contradictoires entre Etats, minorités et diasporas concerne également au MoyenOrient les Kurdes, les Libanais, les Arméniens. Tous ces groupes identitaires sont engagés dans des constructions nationales imparfaites ou dans des affrontements avec des Etats (au moins dans le cas kurde) qui devraient logiquement déboucher sur la mise en place d'un système d'arbitrage régional s'efforçant de concilier la souveraineté des Etats, la protection des minorités et la liberté des individus. On est encore loin d'aboutir à un cadre pouvant satisfaire les besoins de sécurité des uns, le désir de prospérité des autres, la reconnaissance des traumatismes historiques créateurs d'identités collectives et les besoins de mobilité sociale des individus. De tels arbitrages ne pourraient logiquement se faire que dans un ensemble démocratique respectueux des individus comme des minorités. Faute de cadre idéal, l'arbitrage international semble le recours inévitable en cas de crise majeure. Mais les Etats ayant les ressources nécessaires n'ont plus guère la volonté d'en payer le prix, aussi bien en termes monétaires que dans la prise en charge du contrôle de la violence. La construction d'un sous-système régional dépendant peut donc apparaître dans ces conditions comme une solution réaliste. C'est en ce sens que le cas palestinien pourrait, par l'attention internationale qu'il suscite, favoriser la construction de systèmes régionaux de protection et de garanties qui auraient pour but d'éviter une dérive de type balkanique. Son absence se traduirait autrement par des déplacements de populations et des 
violences qui ne sont pas absentes dans la région d'un passé proche lié à la destruction de l'Empire ottoman. La diaspora palestinienne née de la mise en place de l'Autonomie devrait donc favoriser la recherche d'un nouveau type de rapports plus respectueux entre Etats, minorités et diasporas. L'ouvrage de Basma Kodmani-Darwish aura, pour sa part, largement contribué à définir les contours du problème sans limiter son approche dans son effort de conceptualisation à la dimension palestinienne du problème, mais en la situant dans un espace territorial soucieux d'un nouvel équilibre entre les principaux acteurs étatiques ou non qui l'habitent. Jusqu'alors, la création des Etats-nations autoritaires n'a constitué qu'une réponse imparfaite aux bouleversements des années vingt. Leur remise en cause apparaît impossible, mais leur dépassement reste encore à construire sous la forme d'un ensemble régional bénéficiant de soutiens et soumis à un contrôle international.

\section{REMY LEVEAU}

Wilhelm Heitmeyer, Joachim Müller, Helmut Schröder, Verlockender Fundamentalismus, Frankfurt am Main Edition Suhrkamp Verlag, 1997, 277 pages.

9 Verlockender Fundamentalismus (La tentation du fondamentalisme), qui a été sévèrement critiqué par des sociologues allemands, a trouvé un écho largement favorable dans la presse. Cette différence de traitement décèle des enjeux de publication, et en particulier ceux au sujet de l'islam et des jeunes immigrés en Allemagne. En un sens, la lecture de cet ouvrage permet de saisir les représentations allemandes sur les jeunes Turcs musulmans, mais n'apporte que peu d'informations sur les pratiques islamiques et les formes de religiosité. Le livre de Heitmeyer, Müller et Schröder relève certes d'une relative nouveauté dans la sociologie allemande de l'islam et de l'immigration. Il rompt en effet avec les analyses en termes organisationnels sur les fédérations turco-islamiques, jusqu'à présent prédominantes dans l'appréhension de " l'islam transplanté ". Les auteurs fondent leur étude sur une enquête quantitative menée parmi des écoliers âgés de 15 à 21 ans et d'origine turque (1221 personnes interrogées) dans le Land Nordrhein Westfahlen. Leur objectif est d'analyser la religiosité musulmane parmi les adolescents et les jeunes adultes issus de familles immigrées. Cette approche s'inscrit dans la continuité des publications précédentes sur l'islam et sur la deuxième génération des immigrés turcs en Allemagne, dans la mesure où ils choisissent un angle de vue qui place " le fondamentalisme " et la potentialité de conflits " ethno-culturels " (" enthnisch-kulturelle Konflikt-potentiale ") au centre de la problématique. En cela, elle perpétue l'appréhension de la deuxième génération des immigrés turcs en termes ethniques, qui caractérise toute la littérature dans ce domaine. Ici, comme dans la majorité des publications, le Turc, musulman ou pas, né et scolarisé en Allemagne, est " l'autre ", celui qui vient de l'extérieur. Dans cette perspective, il ne fait pas partie de " la culture allemande " et ne peut par conséquent être étudié qu'à travers la notion " ethnique ". La méthode quantitative par questionnaire, choisie par les auteurs, mérite une critique approfondie et une discussion en détail sur les différentes questions posées. Un compte rendu ne permet pas d'aborder l'ensemble des problèmes méthodologiques. Toutefois à plusieurs niveaux, la pertinence de la démarche n'est pas évidente. Les auteurs procèdent, en effet, à une analyse des résultats, en supposant la stabilité des formes de religiosité et des opinions des adolescents et des jeunes adultes d'origine turque. Or, d'autres études montrent l'affaiblissement des institutions religieuses et l'hétérogénéité de références dans l'islam de la diaspora qui conduisent à des constructions fragiles et individualisées 
de prises de positions religieuses, ce que l'enquête par questionnaire menée à un moment donné ne peut révéler. Comme les trois auteurs n'en tiennent pas compte, ils traitent leurs résultats comme un ensemble stable et cohérent. Les intentions, exprimées par les enquêtés dans un contexte donné, sont dès lors considérées comme des dispositions qui conduisent inévitablement à la violence. Les questions à choix multiple enferment, d'ailleurs, l'enquêté dans une conception de l'islam qui renvoie à l'opposition simpliste entre traditionalisme ethnique et sécularisation de l'individu. L'analyse réduit en cela les expressions religieuses en une version supposée " fondamentaliste " et, aux yeux des auteurs, forcément agressive et dangereuse et en une religiosité individuelle, confinée dans l'intimité de la personne. Toutes les autres formes de pratiques religieuses - intermédiaires ou dépassant cette antinomie - sont exclues de la réflexion. Les a priori méthodologiques se transforment en un parti pris explicite des auteurs dans la mesure où ils déclarent n'être concernés que par l'idéologisation de l'islam. En cela, et c'est plus grave, le livre participe à la construction d'une " menace turque " en Allemagne - une représentation qui circule déjà dans l'espace public à travers les débats sur " la criminalité des étrangers " (Ausländerkriminalität), " le terrorisme " importé par les réfugiés politiques, etc. Volens nolens, il sert désormais à légitimer une politique sécuritaire. La religiosité personnelle n'intéresse même pas les auteurs, en dehors du libre exercice du culte que la " société majoritaire " (" Mehrheitsgesellschaft ") devrait garantir (p.36). Le lecteur n'apprend pratiquement rien sur l'utilisation subjective de l'islam pour s'intégrer dans la société allemande, surmonter des conflits générationnels ou dépasser des discriminations dans la vie quotidienne. Heitmeyer, Müller et Schröder définissent le fondamentalisme en tant que mutation de la religion en idéologie politique qui tente de monopoliser la vision du monde et de faire advenir cette dernière par la force. En ce sens, l'islam " fondamentaliste " mène, selon les auteurs, à " un terrain en affinité avec la violence " (" auf gewaltaffines Terrain ") (p. 30-32). Tout le matériel empirique est analysé selon cette seule perspective. Après la lecture, le sentiment s'impose que l'objet de la religiosité musulmane des jeunes d'origine turque se réduit à la seule notion d'idéologisation de la religion. Cet aspect mérite, certes, l'attention des chercheurs. Mais il ne résume en rien la réalité des musulmans en Allemagne. Deux tiers de l'échantillon voient, en effet, selon les auteurs eux-mêmes, dans leur religiosité une affaire privée (p. 122). Si le même nombre de personnes attribue à l'islam un rôle social important et souhaite sa reconnaissance publique à l'instar des autres religions (p.123), on ne peut simplement conclure à la transformation de l'islam en idéologie politique ou à une contradiction, comme les auteurs l'indiquent. Au contraire, les opinions des jeunes d'origine turque témoignent de leur intégration dans la République fédérale. Ils vivent, tout de même, dans une société où les confessions de la majorité ont des voix reconnues et légitimes dans l'espace public. Pourquoi l'expression publique de l'islam mènerait-elle forcément à " un terrain en affinité avec la violence " ? Les auteurs lient les résultats de leur enquête aux problèmes de l'individualisation dans la modernité (pp. 24-27), en s'appuyant sur les travaux théoriques du sociologue Ulrich Beck. Dans cette perspective, le fondamentalisme représente, d'après eux, une double réponse aux évolutions sociales : d'un côté, à la dissolution des groupes identitaires traditionnels, de l'autre, aux expériences de discriminations dans la société allemande (pp. 183-184). L'individu " déstabilisé " aurait recours aux traditions de la génération précédente et compenserait sa fragilité subjective par des positions non nuancées (" dé-différenciées ") (pp. 26 et 106-107). Mais, si le fondamentalisme est une expression de la modernité, 
comme le soulignent les auteurs, il se pose tout de même la question de connaître la stabilité de ces religiosités fondamentalistes ou de ces idéologies politiques. Le jeune homme qui donne aujourd'hui des réponses " fondamentalistes " dans une enquête peut aller le lendemain dans une boîte de nuit pour s'amuser, danser et " draguer ". La modernité peut justement s'exprimer à travers ces recompositions d'éléments traditionnellement contradictoires. De telles réflexions échappent à la démarche des trois chercheurs qui simplifient outrancièrement la thèse de Beck sur la " désintégration sociale ", ce qui les conduit à des conclusions réductrices, uniquement orientées vers le spectaculaire. Cela est d'autant plus étonnant que Heitmeyer était connu en Allemagne pour ses études détaillées et originales sur l'extrême droite et les jeunes néo-nazis. Pour justifier leur position, Heitmeyer, Müller et Schröder insistent sur la spécificité allemande dans laquelle évoluent les jeunes adultes d'origine turque, qui favorise et solidifie les positions " fondamentalistes ". Les expériences de discrimination et le refus gouvernemental de reconnaitre un statut minoritaire aux Turcs contribuent, aux yeux des auteurs, à la constitution " d'une identification ethnoculturelle " qui " figerait " la pratique islamique dans un traditionalisme et un communautarisme ethnique (p. 183). L'islamisation des jeunes est dès lors appréhendée en termes d'une opposition entre majorité et minorité culturelles, d'où l'analyse des auteurs sur " les potentialités pour des conflits ethno-culturels " (p.11). Pour conclure, bien que Heitmeyer, Müller et Schröder tiennent compte d'une manière convaincante des conditions politiques des immigrés (l'accès difficile à la citoyenneté, la mauvaise réputation publique du Turc et la représentation de l'immigré comme étranger), la question se pose de savoir si la religiosité musulmane en Allemagne peut être réduite à une problématique de la violence ou à un phénomène d'ethnicité qui confirmerait son extraterritorialité par rapport à la société allemande. Les auteurs ont certainement réussi à créer un intérêt public pour les évolutions de la minorité germano-turque (pp. 12-13, 193). Mais la religiosité musulmane de la jeunesse d'origine turque reste, même après la lecture, un domaine inconnu, parce que les chercheurs se sont concentrés sur la virtualité d'un comportement violent d'une infime minorité de croyants. La violence, qui existe réellement entre divers groupes politiques de l'immigration turque en Allemagne, n'apparaît, par contre, nullement comme problématique de " La tentation du fondamentalisme ".

\section{NOTES}

1. Kodmani-Darwish (Basma), La diaspora palestinienne, Paris, PUF, 1997, 263 p. 
INDEX

Index géographique : Moyen-Orient, Palestine

Mots-clés : Diaspora, Etat-nation 